\title{
Performance analysis of GPS augmentation using Japanese Quasi-Zenith Satellite System
}

\author{
Falin $\mathrm{Wu}^{1}$, Nobuaki Kubo ${ }^{1}$, and Akio Yasuda ${ }^{1}$ \\ ${ }^{1}$ Tokyo University of Marine Science and Technology, 2-1-6 Etchujima, Koto-ku, Tokyo 135-8533, Japan
}

(Received October 3, 2003; Revised January 7, 2004; Accepted January 7, 2004)

\begin{abstract}
The current GPS satellite constellation provides limited availability and reliability for a country like Japan where mountainous terrain and urban canyons do not allow a clear skyline to the horizon. At present, the Japanese QuasiZenith Satellite System (QZSS) is under investigation through a government-private sector cooperation. QZSS is considered a multi-mission satellite system, as it is able to provide communication, broadcasting and positioning services for mobile users in a specified region with high elevation angle. The performance of a Global Navigation Satellite System (GNSS) can be quantified by availability, accuracy, reliability and integrity. This paper focuses on availability, accuracy and reliability of GPS with and without augmentation using QZSS. The availability, accuracy and reliability of GPS only and augmented GPS using QZSS in the Asia-Pacific and Australian area is studied by software simulation. The simulation results are described by the number of visible satellites as a measure of availability, geometric dilution of precision as a measure of accuracy and minimal detectable bias, and bias-tonoise rate as a measure of reliability, with spatial and temporal variations. It is shown that QZSS does not only improve the availability and accuracy of GPS positioning, but also enhances the reliability of GPS positioning in Japan and its neighboring area.
\end{abstract}

\section{Introduction}

Currently, Japan leads the world in various applications of GPS equipment and services for civil use. About five million GPS-equipped cellular phones are in use, and approximately two million GPS-equipped car navigation units are sold annually in Japan with a cumulative total of about ten million units sold from 1993 to 2002 (Petrovski et al., 2003). The spread of civil use of the GPS service in such areas as car navigation, aviation, maritime, mapping, land surveying, telecommunications and so forth, calls for reliability and availability of the positioning service, which at present has some limitations due to the limited satellite visibility typical in Japan because of its urban canyons and mountainous regions. A GEO-stationary satellite system couldn't meet these requirements because it has an approximate $45^{\circ}$ elevation angle limitation in mid latitude regions. However, the planned Japanese Quasi-Zenith Satellite System (QZSS) will augment GPS to meet these requirements.

QZSS is a constellation consisting of several Highly Elliptic Orbit (HEO) satellites orbiting in different high inclination planes with a GEO-synchronous orbital period. Each satellite is placed on orbit so as to pass over the same ground track at a constant interval. Eccentricity and inclination are selected so that users are able to receive the signal from at least one of the satellites near the zenith direction (i.e. with high elevation angle) at any time. This is the origin of the name Quasi-Zenith Satellite System. Satellite systems like QZSS that utilize a high inclination orbit are indispensable

Copy right $(\mathrm{C}$ The Society of Geomagnetism and Earth, Planetary and Space Sciences (SGEPSS); The Seismological Society of Japan; The Volcanological Society of Japan; The Geodetic Society of Japan; The Japanese Society for Planetary Sciences. for high latitude regions. The Soviet Union (now Russia) has used the Molniya orbit for satellite communications since 1965. For mid latitude regions, although GEO satellite systems have been utilized in the past, some systems have, however, just been implemented for mobile users. Sirius satellite radio has started to provide their Digital Audio Broadcasting (DAB) services for mobile users in North America via three HEO satellites. In Europe, Global Radio is also planning to begin a similar DAB service in a couple of years using a similar HEO satellite system (Kogure and Kawano, 2003).

In this paper we focus on the performance of GPS augmentation using the Japanese QZSS. The constellation and signal structure of QZSS will be briefly reviewed in Section 2 . The three single baseline models and stochastic model of GPS augmentation using QZSS will be analyzed in Section 3. The measures for performance analysis will be presented in Section 4. The performance of the GPS augmentation using QZSS will be shown in terms of the Number of Visible Satellites (NVS), Geometric Dilution Of Precision (GDOP), Minimal Detectable Bias (MDB) and Bias-toNoise Rate (BNR) with spatial and temporal variations in Section 5.

\section{Japanese Quasi-Zenith Satellite System}

QZSS is a new concept developed by the private sector, with the government sector assuming responsibility for the associated technology development, and especially the portion of the project concerned with the positioning service. This effort has taken place in the context of Japan-U.S. cooperation in GPS, formalized by the GPS Joint Statement signed on November 22, 1998. The 1998 policy statement established a cooperative mechanism that provided for an- 
Table 1. Parameters of the three QZSS satellite constellation options.

\begin{tabular}{cccccc}
\hline QZSS option & Ground track & Satellite number & Eccentricity & Inclination & Semi-major axis \\
\hline 1 & Asymmetrical 8-shape & 3 & 0.099 & $45.0^{\circ}$ & $42,164 \mathrm{~km}$ \\
2 & Egg-shape & 3 & 0.360 & $52.6^{\circ}$ & $42,164 \mathrm{~km}$ \\
3 & Symmetrical 8-shape & 3 & 0.000 & $45.0^{\circ}$ & $42,164 \mathrm{~km}$ \\
\hline
\end{tabular}

(a) Option 1

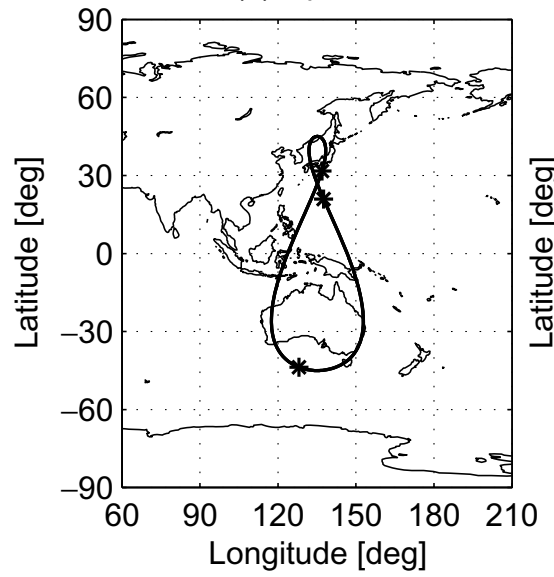

(b) Option 2

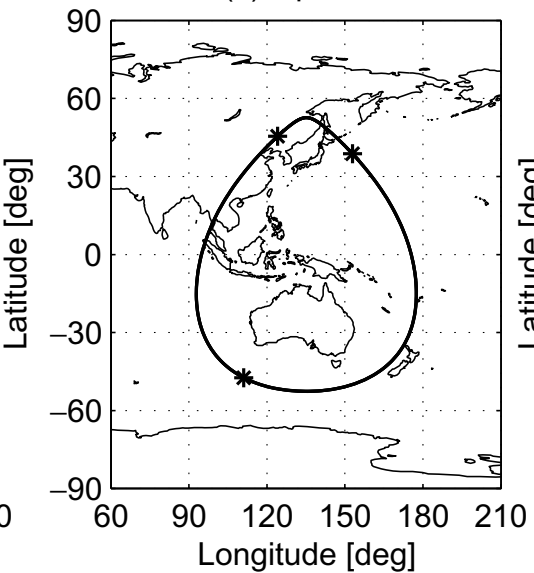

(c) Option 3

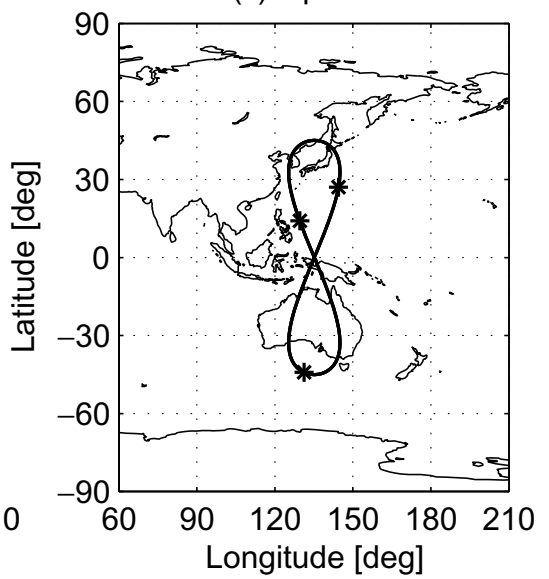

Fig. 1. Ground tracks of the three QZSS satellite constellation options.

nual plenary meetings and working groups. Japan's stated policy objective is "to secure and enhance user interest", and the QZSS initiative is a logical outcome of this policy (Petrovski et al., 2003).

\subsection{Satellite constellation}

Five types of constellations that are being considered for QZSS were registered with the International Telecommunications Union in November 2002 (Petrovski et al., 2003). It is yet to be decided which satellite constellation will be selected for the QZSS, because the investigations are still under way. Table 1 summarizes the main characteristics of the three most favored satellite constellations that will be investigated in this study. Each satellite constellation option is composed of three satellites on orbit and one spare satellite on the ground. The semi-major axis of all three satellite constellations is $42,164 \mathrm{~km}$. Different eccentricity and inclination are selected for the three satellite constellations. Figure 1 shows the ground tracks of the three satellite constellations. The eccentricities of the three satellite constellations are approximately $0.099,0.360$ and 0.000 . Inclinations of the three satellite constellations are approximately $45.0^{\circ}$, 52.6 $6^{\circ}$ and $45.0^{\circ}$ (Kogure and Kawano, 2003; Kon, 2003).

2.1.1 QZSS option 1 With eccentricity 0.099 and inclination $45.0^{\circ}$, the ground track of the satellite constellation scribes an asymmetrical figure 8-shape. This satellite constellation option focuses on the benefit for mobile communication users with tracking antenna and feeder link stations in Japan. One advantage of this satellite constellation is that various services, such as communication, broadcasting and positioning, will be available equally to users in Japan and neighboring countries.

2.1.2 QZSS option 2 With eccentricity 0.360 and inclination $52.6^{\circ}$, the ground track of the satellite constellation scribes an egg-shape figure. The advantage of this satellite constellation option is that broadcasting related services will be provided a little more effectively for users in Japan and its neighboring countries than in the case of the two other satellite constellation options.

2.1.3 QZSS option 3 With eccentricity 0.000 and inclination $45.0^{\circ}$, the ground track of the satellite constellation scribes a symmetrical figure 8-shape centred on the equator, and users in both hemispheres can receive services equally effectively. But this satellite constellation option has to maneuver the satellite frequently to avoid collisions as a satellite passes through the highly populated geostationary satellite belt. In addition this satellite constellation would provide less favorable visibility over the northern hemisphere compared with the two other satellite constellation options.

Figure 2 shows the temporal variations of elevation for the three QZSS satellite constellations at Tokyo. It is shown that a user can track at least one QZSS satellite with $70^{\circ}$ mask elevation, and two QZSS satellites with $30^{\circ}$ mask elevation for each of the three QZSS satellite constellation.

Further information about the QZSS satellite constellations can be found in Murotani et al. (2003), Kawano (1999), Takahashi et al. (1999), Kimura and Tanaka (2000), Kawano, (2001), and Yamamoto and Kimura (2003).

\subsection{Signal structure}

At the GPS-QZSS Technical Working Group meeting in early December 2002, Japanese and U.S. government representatives discussed the creation of QZSS. The representatives from the two nations deliberated the technical requirements for the QZSS signal structure, codes and power. To date, the positioning service of QZSS is considered to be an advanced space augmentation system for GPS. QZSS will use the same signal structure as GPS, and employ pseudorandom noise (PRN) code which used by the GPS constellation and WAAS. Other types of signal modulation are also un- 
(a) QZSS option 1

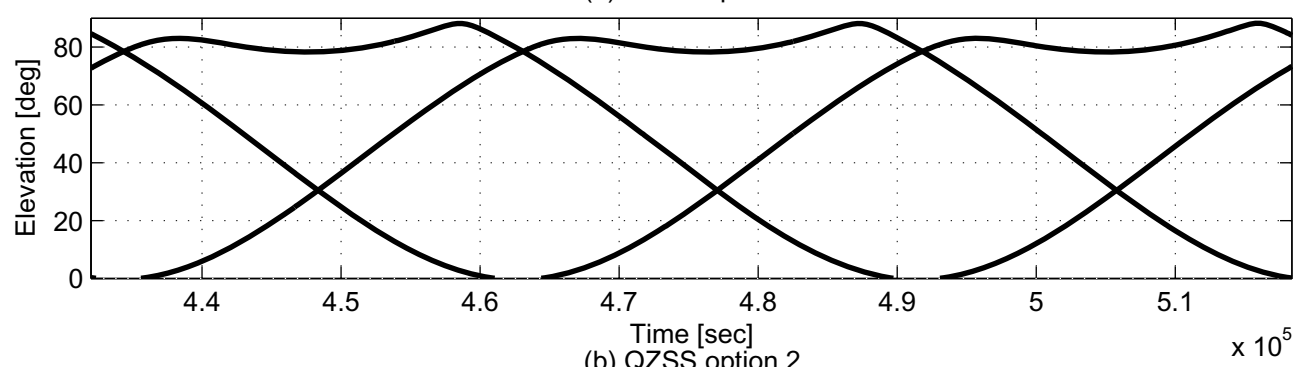

(b) QZSS option 2

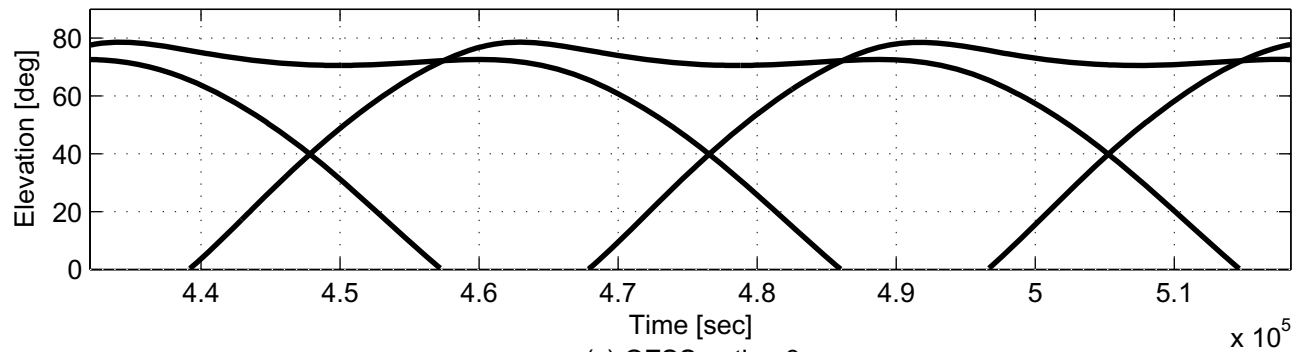

(c) QZSS option 3

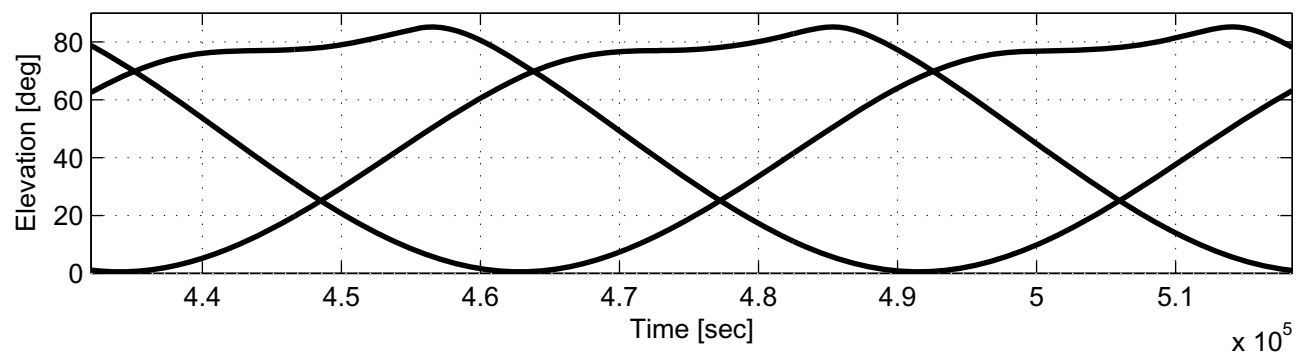

Fig. 2. Elevation temporal variations of the three QZSS satellite constellations at Tokyo.

Table 2. Possible signals of GPS and QZSS, with corresponding frequencies, wavelengths and typical code measurement accuracy.

\begin{tabular}{cccc}
\hline Signal & $\begin{array}{c}\text { Frequency } \\
{[\mathrm{MHz}]}\end{array}$ & $\begin{array}{c}\text { Wavelength } \\
{[\mathrm{m}]}\end{array}$ & $\begin{array}{c}\sigma_{\text {code }} \\
{[\mathrm{m}]}\end{array}$ \\
\hline L1 & 1575.42 & 0.1903 & 0.30 \\
L2 & 1227.60 & 0.2442 & 0.30 \\
L5 & 1176.45 & 0.2548 & 0.10 \\
\hline
\end{tabular}

der consideration. Currently, the governmental institutions involved continue to work towards a definition of the signal structure. At the time of writing of this paper the latest meeting had been held in May, 2003 (Petrovski et al., 2003; Kogure and Kawano, 2003).

Table 2 gives an overview of possible GPS and QZSS signals, with corresponding frequencies, wavelengths and typical code measurement accuracy (Shaw et al., 2002; Teunissen et al., 2002; Verhagen, 2002b) that are used in this study.

\section{GPS Augmentation Using QZSS}

The measured ranges of GPS and QZSS, by pseudorange and carrier phase respectively, are related to the unknown parameters via the following generic measurement equations (Tiberius et al., 2002),

$$
P_{r, i}^{s}=\rho_{r}^{s}+d_{r, i}-d^{s, i}+\frac{f_{L 1}^{2}}{f_{i}^{2}} I_{r}^{s}+T_{r}^{s}+e_{r, i}^{s}
$$

$$
\begin{aligned}
\Phi_{r, i}^{s}= & \rho_{r}^{s}+\delta_{r, i}-\delta^{s, i}-\frac{f_{L 1}^{2}}{f_{i}^{2}} I_{r}^{s}+T_{r}^{s} \\
& +\lambda_{i} N_{r, i}^{s}+\varepsilon_{r, i}^{s}
\end{aligned}
$$

where $\Phi$ and $P$ are the carrier phase and pseudorange, respectively. $\rho$ is the geometric range from satellite $s$ to receiver $r ; i$ is the L-band frequency signals of GPS and QZSS, $i=L 1, L 2$ and $L 5 . f$ is the frequency of the signal. $I$ is the ionospheric delay on L1 frequency and $T$ is the tropospheric delay. $d$ and $\delta$ are the clock error for code and carrier phase observations, respectively. $\lambda$ and $N$ are the wavelength and cycle ambiguity number of signal $i$ carrier phase. $\varepsilon$ and $e$ represent the effect of receiver noise on the carrier phase and the pseudorange, respectively.

In this study, the three single baseline models that will be considered are: geometry-free (GF) model, rovingreceiver geometry-based (RR) model and the stationaryreceiver geometry-based (SR) model (Teunissen, 1997; Teunissen, 1998; Teunissen and Jong, 1998; de Jong, 2000; Verhagen, 2002b).

\subsection{Three single baseline models}

From Eq. (1) and (2), the observations, with or without parameterization in terms of the baseline components, are collected by type, the code and phase observations on all frequencies. Then the three single baseline models for $k$ epochs of data can all be written in a generic form (Verhagen, 2002b),

$$
y=\left[I_{k} \otimes M e_{k} \otimes N\right]\left[\begin{array}{l}
b \\
a
\end{array}\right]+n
$$


where $a$ is the component of ambiguities, $\otimes$ is Kronecker product, and $\underset{p \times q}{M} \otimes N=\left[\begin{array}{ccc}m_{11} N & \cdots & m_{1 q} N \\ \vdots & \ddots & \vdots \\ m_{p 1} N & \cdots & m_{p q} N\end{array}\right]$. The notation $I_{k}$ denotes a identity matrix of order $k$. Different models have different matrices $M, N$ and unknowns $b$.

For the GF model, the observation equation isn't parameterized in terms of the baseline components, but remains parameterized in terms of the unknown double differenced receiver-satellite ranges. The unknowns $b$ are simply the ranges $\rho$, and design matrices are given by,

$$
M=e_{2 \zeta} \otimes I_{m-1} ; \quad N=C_{2} \otimes I_{m-1}
$$

where $\zeta$ is the observed frequency number, $m$ is the number of observed satellites, $C_{2}=c_{2} \otimes I_{\zeta}$. The notations $c_{j}$ and $e_{j}$ denote a vector with a one at the $j$-th entry and zeros otherwise, and a vector consisting of $j$ ones, respectively.

In case of the geometry-based model, the observation equations should be parameterized in terms of the baseline components. A linearization of the double differenced receiver-satellite geometry ranges with respect to the baseline components are

$$
\Delta \rho_{k}=\bar{G}_{k} \Delta b_{k} \quad \text { with } \quad \bar{G}_{k}=\left[\frac{\partial \rho_{k}}{\partial b_{k}}\right]
$$

where $b_{k}$ and $\Delta b_{k}$ are the baseline vector and its increment of epoch $k$, and $\bar{G}_{k}$ is the $(m-1) \times 3$ matrix that captures the receiver-satellite geometry, respectively. The elements of the matrix, $\bar{G}_{k}$, are time-dependent. However, because of the slowly changing geometry it is considered time-invariant here, i.e. $\bar{G}_{k}=\bar{G}=$ constant.

For the RR model, the unknowns $b$ are the $3 k$ baseline increments $\Delta b_{k}$ (three for each epoch), and the design matrices are given by,

$$
M=e_{2 \zeta} \otimes \bar{G} ; \quad N=C_{2} \otimes I_{m-1} .
$$

For the SR model, the $k$ baselines $b_{k}$ collapse to one single baseline $b$, the unknowns $b$ are the 3 baseline increments $\Delta b$, and the design matrices are given by,

$$
M=\emptyset ; \quad N=\left(e_{2 \zeta} \otimes \bar{G}, C_{2} \otimes I_{m-1}\right) .
$$

The three single baseline models given here can be applied to either GPS or augmented GPS using QZSS,

$$
\begin{aligned}
y_{G P S} & =\left[I_{k} \otimes M_{G P S} e_{k} \otimes N_{G P S}\right]\left[\begin{array}{c}
b \\
a_{G P S}
\end{array}\right]+n \\
y_{Q Z S S} & =\left[I_{k} \otimes M_{Q Z S S} e_{k} \otimes N_{Q Z S S}\right]\left[\begin{array}{c}
b \\
a_{Q Z S S}
\end{array}\right]+n
\end{aligned}
$$

where, the subscript GPS and QZSS denote GPS only and augmented GPS using QZSS, respectively.

\subsection{Stochastic model}

It is assumed that the variance-covariance matrix of the single differenced observations of one satellite, without elimination of the ionospheric parameters, is given by,

$$
C_{P \Phi}=\left[\begin{array}{ll}
C_{P} & \\
& C_{\Phi}
\end{array}\right]
$$

where $C_{P}$ and $C_{\Phi}$ are the variance-covariance matrices of the code and phase observations, respectively. So, there may be correlations between the code observations and between the phase observations on different frequencies. Since the ionospheric parameters were eliminated from the measurement equations, the variance-covariance matrix becomes,

$$
C=C_{P \Phi}+2 s^{2}\left[\begin{array}{c}
\mu \\
-\mu
\end{array}\right]\left[\begin{array}{c}
\mu \\
-\mu
\end{array}\right]^{T}
$$

where $\mu=\left(\frac{f_{L 1}^{2}}{f_{i}^{2}}\right)^{T}, s^{2}$ is the undifferenced ionospheric weighing factor in units of square meters.

The complete double differenced variance-covariance matrix becomes (Verhagen, 2002b),

$$
Q_{y}=I_{k} \otimes C \otimes E
$$

where $E=D^{T} D$, and $D^{T}$ is the $(m-1) \times m$ double differencing operator. No satellite-dependent weighting is applied.

The variance-covariance matrix that corresponds to the measurement models of GPS only and augmented GPS using QZSS, given in Eqs. (8) and (9), become,

$$
\begin{aligned}
Q_{y_{G P S}} & =I_{k} \otimes C_{G P S} \otimes E_{G P S} \\
Q_{y_{Q Z S S}} & =I_{k} \otimes C_{Q Z S S} \otimes E_{Q Z S S} .
\end{aligned}
$$

\section{Performance Measures for GPS Augmentation Using QZSS}

Availability, accuracy, reliability and integrity are often used as measures to quantify the performance of a navigation system (O'Keefe, 2001). In the context of GNSS, availability usually refers to the number of satellites or other ranging signals available to the user. From a generic radio navigation standpoint, availability refers to the percentage of the time that a system is able to provide the user with navigation solutions (Kaplan, 1996). Accuracy is a measure of how close the navigation solution provided by the system is to the user's true location and velocity. Generally, the accuracy of a system can be decomposed into two quantities: User Equivalent Range Error (UERE) and GDOP. UERE is obtained by mapping all of the system and user errors into a single error in one user measured range. GDOP is the satellite geometry dependent quantity that maps the UERE (an error in observation space) into a user accuracy (in position space). Reliability is defined as one minus the probability of system failure (FRP, 1999). Reliability can be subdivided into internal reliability and external reliability. Integrity is defined as "the ability of a system to provide timely warnings to users when the system should not be used for navigation" (FRP, 1999; Kaplan, 1996).

In this section, internal reliability, represented by MDB, and external reliability, represented by MDE or BNR (Verhagen, 2002b), will be introduced. In this study, the quantities of NVS, GDOP, MDB and BNR will be used to evaluate the performance of GPS augmentation using QZSS.

\subsection{Internal reliability}

The MDB describes the minimum model error that can be detected by using the appropriate test statistics (Teunissen, 1997, 1998; de Jong, 2000; O’Keefe, 2001; Verhagen, 
2002b; Verhagen and Joosten, 2003). The MDB can be computed once the type of model error is specified, so that the null-hypothesis $\left(H_{0}\right)$, which assumes that there is no error, can be tested against the alternative hypothesis $\left(H_{a}\right)$, which assumes the presence of the error. The two hypotheses are defined as (Teunissen, 1998)

$$
\begin{array}{llrl}
H_{0}: E\{y\} & =A x, & D\{y\} & =Q_{y} \\
H_{a}: E\{y\} & =A x+c \nabla, & D\{y\} & =Q_{y}
\end{array}
$$

where $E\{\cdot\}$ and $D\{\cdot\}$ are the expectation and dispersion operators, respectively, $y$ is the $p$-vector observations, $A$ is the $p \times q$ design matrix, $x$ is the $q$-vector of unknown parameters, $c$ is a known $p$-vector which specifies the type of model error, and $\nabla$ is its unknown size, and $Q_{y}$ is the variance matrix of the observations.

The uniformly most powerful test statistic for testing $H_{0}$ against $H_{a}$ is given as (Teunissen, 1998)

$$
T=\frac{\left(c^{T} Q_{y}^{-1} \mathcal{P}_{A}^{\perp} y\right)^{2}}{c^{T} Q_{y}^{-1} \mathcal{P}_{A}^{\perp} c}
$$

where $\mathcal{P}_{A}^{\perp}=I_{p}-\mathcal{P}_{A}$, and $\mathcal{P}_{A}$ is the orthogonal projector on the range space of $A, \mathcal{P}_{A}=A\left(A^{T} Q_{y}^{-1} A\right)^{-1} A^{T} Q_{y}^{-1}$. The test statistic $T$ has the following Chi-squared distributions under $H_{0}$ and $H_{a}$

$$
H_{0}: T \sim \chi^{2}(1,0) ; \quad H_{a}: T \sim \chi^{2}(1, \lambda)
$$

where $\lambda$ is the non-centrality parameter,

$$
\lambda=\nabla^{2} c^{T} Q_{y}^{-1} \mathcal{P}_{A}^{\perp} c .
$$

The non-centrality parameter $\lambda$ can be computed when reference values are chosen for the level of confidence $\alpha_{0}$ (the probability of rejecting $H_{0}$ when it is true) and the detection power $\gamma_{0}$ (the probability of rejecting $H_{0}$ when $H_{a}$ is true). Once the parameter $\lambda_{0}=\lambda\left(\alpha_{0}, \gamma_{0}\right)$ is known, the corresponding size of the bias, MDB, that can just be detected, follows from Eq. (19) as (Teunissen, 1998)

$$
|\nabla|=\sqrt{\frac{\lambda_{0}}{c^{T} Q_{y}^{-1} \mathcal{P}_{A}^{\perp} c}}
$$

where $\mathcal{P}_{A}^{\perp}=I_{p}-A\left(A^{T} Q_{y}^{-1} A\right)^{-1} A^{T} Q_{y}^{-1}$.

Potential model errors in GNSS applications are outliers in the code observations, and/or cycle slips in the carrier phase observations. The three single baseline models described in Section 3.1 can be used to find expressions for the corresponding MDB using Eq. (20.) The design matrix $A$ of the three single baseline models follows from the results of Section 3.1 as

$$
A=\left[M_{k} N_{k}\right] \text { with } M_{k}=I_{k} \otimes M, N_{k}=e_{k} \otimes N
$$

where

$$
\left\{\begin{array}{ll}
M=e_{2 \zeta} \otimes I_{m-1}, N=C_{2} \otimes I_{m-1} & \mathrm{GF} \\
M=e_{2 \zeta} \otimes \bar{G}, N=C_{2} \otimes I_{m-1} & \mathrm{RR} \\
M=\emptyset, N=\left(e_{2 \zeta} \otimes \bar{G}, C_{2} \otimes I_{m-1}\right) & \mathrm{SR}
\end{array} .\right.
$$

It is assumed that the error occurs in the observation on frequency $i$ at epoch $l$ in the double differenced range to satellite $s \in(1, \cdots, m)$, so that the vector $c$ in Eq. (20) becomes

$$
\left.\left.c=\begin{array}{l}
c_{l} \\
s_{l}
\end{array}\right\} \otimes d, \text { with } d=\begin{array}{l}
c_{i} \\
c_{i+\zeta}
\end{array}\right\} \otimes d_{s} \begin{aligned}
& \text { code outlier } \\
& \text { cycle slip. }
\end{aligned}
$$

The vector $s_{l}$ contains zeros as the first $(l-1)$ entries, and ones as the last $v=(k-l+1)$ entries, i.e. $v$ is the length of the slip. Recall that $\zeta$ is the frequency number. The vector $d_{s}$ is the $s$-th column of the matrix $D^{T}$.

Using the properties of the Kronecker product and orthogonal projectors, it was shown in Teunissen (1998), Verhagen (2002b) and Verhagen and Joosten (2003) that the MDB for a code outlier $\left|\nabla_{P}\right|$ and a cycle slip $\left|\nabla_{\Phi}\right|$, respectively, are given by

$$
\begin{aligned}
\left|\nabla_{P}\right|= & \left(\lambda _ { 0 } \left(d^{T} Q_{y}^{-1} d-\left(1-\frac{1}{k}\right) d^{T} Q_{y}^{-1} \mathcal{P}_{M} d\right.\right. \\
& \left.\left.-\frac{1}{k} d^{T} Q_{y}^{-1}\left(\mathcal{P}_{N}+\mathcal{P}_{\mathcal{P}_{N} M}\right) d\right)^{-1}\right)^{\frac{1}{2}} \\
\left|\nabla_{\Phi}\right|= & \left(\lambda _ { 0 } v ^ { - 1 } \left(d^{T} Q_{y}^{-1} d-\left(1-\frac{v}{k}\right) d^{T} Q_{y}^{-1} \mathcal{P}_{M} d\right.\right. \\
& \left.\left.-\frac{v}{k} d^{T} Q_{y}^{-1} \mathcal{P}_{N} d\right)^{-1}\right)^{\frac{1}{2}}
\end{aligned}
$$

where

$$
\begin{gathered}
d^{T} Q_{y}^{-1} d=\left(c_{i}^{T} C^{-1} c_{i}\right)\left(d_{s}^{T} E^{-1} d_{s}\right) \\
d^{T} Q_{y}^{-1} \mathcal{P}_{M} d=\left(c_{i}^{T} C^{-1} \mathcal{P}_{e_{2 \zeta}} c_{i}\right) \begin{cases}\left(d_{s}^{T} E^{-1} d_{s}\right) & \mathrm{GF} \\
\left(d_{s}^{T} E^{-1} \mathcal{P}_{\bar{G}} d_{s}\right) & \mathrm{RR} \\
0 & \mathrm{SR}\end{cases} \\
d^{T} Q_{y}^{-1} \mathcal{P}_{N} d=\left(c_{i}^{T} C^{-1} \mathcal{P}_{C_{2}} c_{i}\right)\left(d_{s}^{T} E^{-1} d_{s}\right) \\
d^{T} Q_{y}^{-1} \mathcal{P}_{\mathcal{P}_{N}^{\perp} M} d \\
=\left(c_{i}^{T} C^{-1} \mathcal{P}_{\mathcal{P}_{C_{2}}^{\perp} e_{2 \zeta}} c_{i}\right) \begin{cases}\left(d_{s}^{T} E^{-1} d_{s}\right) & \mathrm{GF} \\
\left(d_{s}^{T} E^{-1} \mathcal{P}_{\bar{G}} d_{s}\right) & \mathrm{RR} \\
\left(d_{s}^{T} E^{-1} \mathcal{P}_{\bar{G}} d_{s}\right) & \mathrm{SR}\end{cases}
\end{gathered}
$$

and, for a cycle slip $c_{i}=c_{i+\zeta}$,

$$
\begin{aligned}
d_{s}^{T} E^{-1} d_{s} & =\frac{m-1}{m} \\
d_{s}^{T} E^{-1} \mathcal{P}_{\bar{G}} d_{s} & =d_{s}^{T} E^{-1} d_{s}-c_{s}^{T} \mathcal{P}_{\left[G e_{m}\right]} c_{s}
\end{aligned}
$$

where $G$ is the design matrix that contains the single differenced receiver-satellite geometry.

The MDBs of GPS only and augmented GPS using QZSS can be computed using Eqs. (24) and (25) with different parameters $y, A$ and $Q_{y}$.

Further information about internal reliability can be found in Teunissen (1997), Teunissen (1998), Teunissen and Kleusberg (1998), Teunissen and de Jong (1998), de Jong (2000), O'Keefe (2001), Verhagen (2002a), Verhagen (2002b), and Verhagen and Joosten (2003).

\subsection{External Reliability}

The MDB gives a measure of the size of the error in the observations that can be detected. A user, however, may 
Table 3. Configuration of all scenarios considered in the simulations.

\begin{tabular}{|c|c|c|}
\hline \multicolumn{2}{|c|}{ System } & GPS, GPS+QZSS (three options) \\
\hline \multicolumn{2}{|c|}{ Baseline model } & Single medium length baseline $(20 \mathrm{~km})$ RR model \\
\hline \multicolumn{2}{|c|}{ Code standard deviation } & $\sigma_{P}=0.300 \mathrm{~m}$ \\
\hline \multicolumn{2}{|c|}{ Phase standard deviation } & $\sigma_{\Phi}=0.003 \mathrm{~m}$ \\
\hline \multicolumn{2}{|c|}{ Ionospheric delay } & $\sigma_{I}=0.020 \mathrm{~m}$ \\
\hline \multicolumn{2}{|c|}{ Tropospheric delay } & $\sigma_{T}=0.010 \mathrm{~m}$ \\
\hline \multicolumn{2}{|c|}{ Mask elevation } & $30^{\circ}$ \\
\hline \multirow[t]{2}{*}{ Spatial simulation } & Date and time & Sep. $5,2003,12: 00$ \\
\hline & Location & $\begin{array}{l}\text { Asia-Pacific, Australian area (Lat: } 90^{\circ} \mathrm{S}-90^{\circ} \mathrm{N} \text {, Lon: } \\
60^{\circ}-210^{\circ} \text { ) }\end{array}$ \\
\hline \multirow[t]{2}{*}{ Temporal simulation } & Date and time & Aug. 31, 2003, 00:00-Sep. 6, 2003, 24:00 \\
\hline & Location & Tokyo $\left(35^{\circ} 39^{\prime} 59^{\prime \prime} \mathrm{N}, 139^{\circ} 47^{\prime} 32^{\prime \prime} \mathrm{E}\right)$ \\
\hline \multirow[t]{2}{*}{ Output } & Spatial variation & NVS, GDOP, MDB and BNR \\
\hline & Temporal variation & NVS, GDOP, MDB and BNR \\
\hline
\end{tabular}

Table 4. Spatial variations of NVS, GDOP, MDB and BNR for L1 code outlier for different constellations (Sep. 5, 2003, 12:00).

\begin{tabular}{|c|c|c|c|c|c|c|}
\hline \multicolumn{3}{|c|}{ System } & \multirow{2}{*}{$\frac{\text { GPS only }}{85.25 \%}$} & \multirow{2}{*}{$\frac{\text { GPS+QZSS } 1}{94.18 \%}$} & \multirow{2}{*}{$\frac{\text { GPS+QZSS } 2}{89.97 \%}$} & \multirow{2}{*}{$\frac{\mathrm{GPS}+\mathrm{QZSS} 3}{95.18 \%}$} \\
\hline Whole area & $\mathrm{N}$ & & & & & \\
\hline \multirow{12}{*}{$\begin{array}{c}\text { Positioning } \\
\text { available area } \\
\text { (GPS only) }\end{array}$} & & MIN & 4 & 4 & 4 & 4 \\
\hline & NVS & MAX & 8 & 9 & 9 & 9 \\
\hline & & MEAN & 5.03 & 6.20 & 6.18 & 6.20 \\
\hline & & MIN & 3.00 & 2.72 & 2.56 & 2.70 \\
\hline & GDOP & MAX & 927.50 & 927.50 & 927.50 & 927.50 \\
\hline & & MEAN & 21.03 & 11.90 & 16.47 & 11.59 \\
\hline & MDB & MIN & 2.21 & 2.16 & 2.15 & 2.15 \\
\hline & (L1 code & MAX & 2.86 & 2.86 & 2.86 & 2.86 \\
\hline & outlier) & MEAN & 2.72 & 2.57 & 2.59 & 2.58 \\
\hline & BNR & MIN & 2.59 & 2.42 & 2.38 & 2.40 \\
\hline & (L1 code & MAX & 4.13 & 4.13 & 4.13 & 4.13 \\
\hline & outlier) & MEAN & 3.93 & 3.64 & 3.66 & 3.67 \\
\hline
\end{tabular}

be more interested in the impact of such an error on his unknown parameters. This is referred to as the external reliability. The external reliability may be represented by the minimal detectable effect (MDE) or the bias-to-noise ratio (BNR) (Verhagen, 2002b).

The MDE is a vector that describes the impact of an MDBsized bias in the observations, $c \nabla$, on each of the unknown parameter to be estimated. The MDE could be derived from Eqs. (15) and (16) (Verhagen, 2002b; Verhagen and Joosten, 2003),

$$
\nabla \hat{x}=\left(A^{T} Q_{y}^{-1} A\right) A^{T} Q_{y}^{-1} c \nabla=Q_{\hat{x}} A^{T} Q_{y}^{-1} c \nabla .
$$

The BNR is a dimensionless measure of the size of the bias in the final estimates with respect to the noise. The BNR is defined as the square root of $\lambda_{\hat{x}}$ (Verhagen, 2002b),

$$
\lambda_{\hat{x}}=\|\nabla \hat{x}\|_{Q_{\hat{x}}}^{2}=(\nabla \hat{x})^{T} Q_{\hat{x}}^{-1}(\nabla \hat{x}) .
$$

For GPS only and augmented GPS using QZSS models, the BNR of the unknown baseline coordinates can be computed by subtracting the contribution of the ambiguities (Verhagen, 2002b),

$$
\lambda_{\hat{b}}=v \cdot \nabla^{2}\left(d^{T} Q_{y}^{-1} d-\frac{v}{k} d^{T} Q_{y}^{-1} \mathcal{P}_{N} d\right)-\lambda_{0}
$$

where, for a code outlier $v=1$.

Further information about external reliability can be found in Verhagen (2002b), and Verhagen and Joosten (2003).

\section{Performance Analysis}

In this section the expected performance of GPS augmentation using the Japanese QZSS is studied by software simulations in terms of the spatial variations as well as the temporal variations of NVS, GDOP, MDB and BNR for L1 code outlier.

Two simulations, spatial simulation and temporal simulation, were conducted. Table 3 gives a summary of all scenarios considered in the simulations. Because a user may be more interested in the unknown baseline coordinates than the 
(a) GPS only

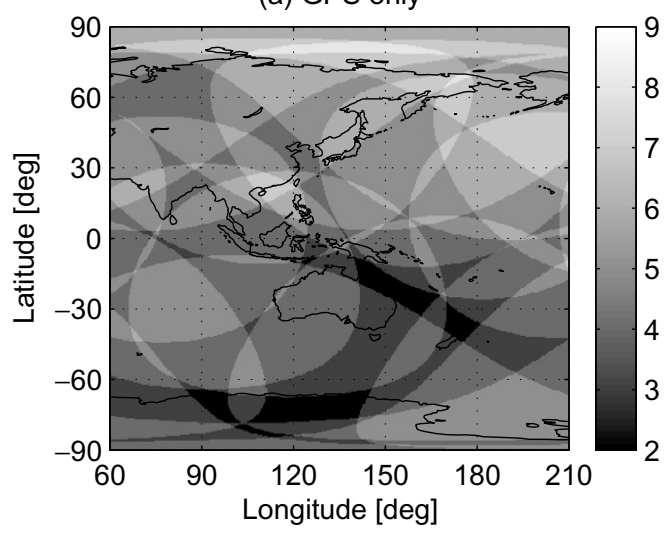

(c) GPS and QZSS option 2

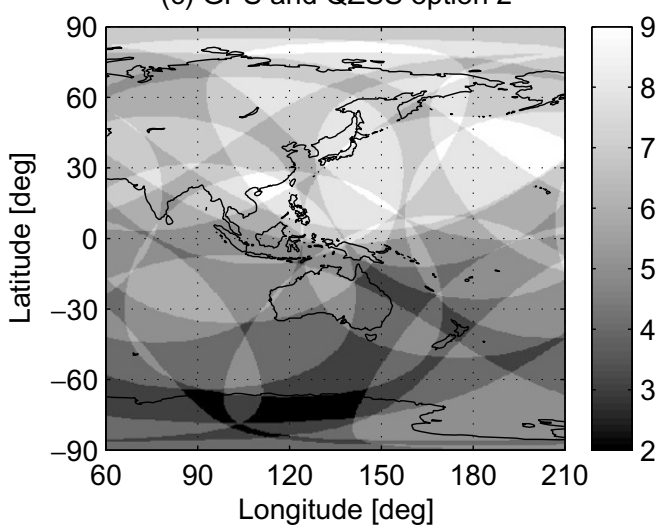

(b) GPS and QZSS option 1

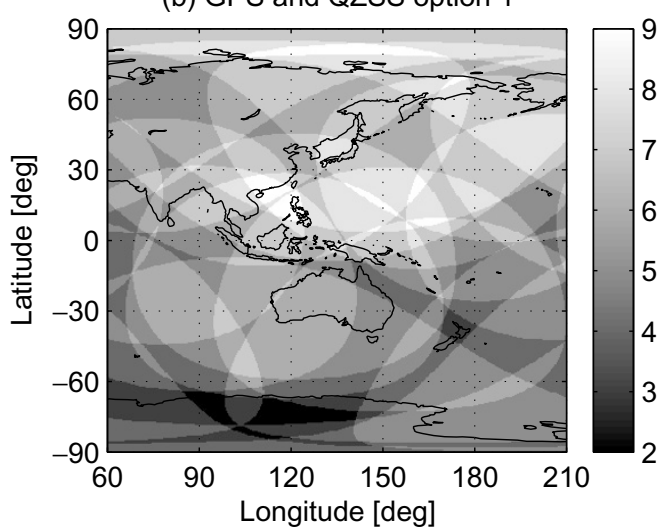

(d) GPS and QZSS option 3

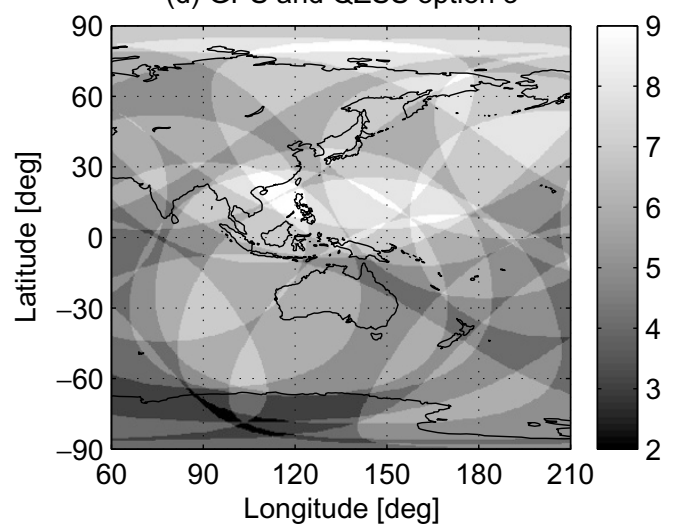

Fig. 3. Spatial variations of NVS for different constellations.

receiver-satellite ranges and the SR model is a typical form of the RR model, only a single medium length baseline (20 $\mathrm{km}) \mathrm{RR}$ model was considered in the simulations. The accuracies of all code and carrier phase observation were set at standard deviation $0.300 \mathrm{~m}$ and $0.003 \mathrm{~m}$, respectively. Ionospheric slant delays and tropospheric zenith delay and were included as unknown parameters, but the uncertainty in these parameters' values had been restricted. Variations in the delays were tolerated to a reasonable small extent on a medium length baseline $\left(\sigma_{I}=0.020 \mathrm{~m}\right.$ and $\left.\sigma_{T}=0.010 \mathrm{~m}\right)$. It has been shown in Section 2.1 that a user at Tokyo can track at least two QZSS satellites with $30^{\circ}$ mask elevation, the visible satellites were masked by a $30^{\circ}$ elevation angle cutoff in the simulations. To compute the positions of the GPS satellites and to simulate the positions of the QZSS satellites, a YUMA almanac was used. The locations of twenty eight GPS satellite and the three QZSS options were continuously simulated for Sep. 5, 2003, 12:00 for spatial simulation and from Aug. 31, 2003, 00:00 to Sep. 6, 2003, 24:00, with a sampling interval of 120 seconds, for temporal simulation. The receiversatellite geometries were simulated in the Asia-Pacific, Australian and New Zealand area (Latitude: $90^{\circ} \mathrm{S}-90^{\circ} \mathrm{N}$, Longitude: $60^{\circ}-210^{\circ}$ ), with a sampling grid of $0.4^{\circ} \times 0.4^{\circ}$, for spatial simulation, and in Tokyo $\left(35^{\circ} 39^{\prime} 59^{\prime \prime} N, 139^{\circ} 47^{\prime} 32^{\prime \prime} E\right)$ for temporal simulation. The spatial and temporal simulations outputted the spatial variations as well as the temporal variations of NVS, GDOP, MDB and BNR for L1 code outlier.

\subsection{Spatial variations}

Before considering temporal variations performance of GPS augmentation using QZSS, the spatial variations performances are analyzed.

Table 4 summarizes the spatial variations of NVS, GDOP, MDB and BNR for L1 code outlier in the case of GPS only and augmented GPS using the three QZSS options at September 5, 2003, 12:00. It is shown that with the augmentation by the three QZSS options, the area where positioning is available (NVS $\geq 4$ ) will be extended from $85.25 \%$ to $94.18 \%, 89.97 \%$ and $95.18 \%$, respectively for each constellation. For spatial variations, augmentation using the three QZSS options can extend the positioning available area, but also enables some locations that have a very high GDOP, MDB and BNR. To analyze the performance of GPS augmentation using the three QZSS options, only the area where positioning is available in the case of GPS only is considered in this subsection.

Figure 3 shows the NVS of GPS only and augmented GPS using the three QZSS options as a function of geographic location. The maximum NVS of GPS only is 8 , but augmented GPS using the three QZSS options give values 9 for all cases. The average NVS of GPS only is about 5.03, but the values of augmented GPS using the three QZSS options are about $6.20,6.18$ and 6.20 , respectively.

Figure 4 shows the spatial variations of GDOP for the GPS only and augmented GPS using the three QZSS options. The minimum GDOP of GPS only is about 3.00, but augmented GPS using the three QZSS options give values 2.72, 2.56 and 
(a) GPS only

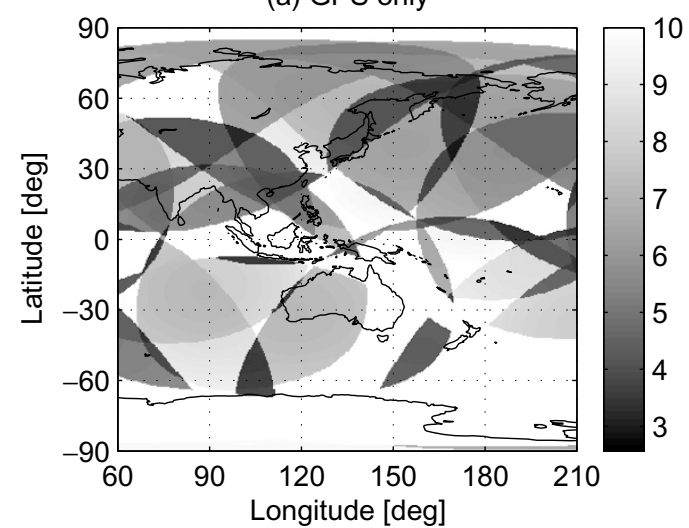

(c) GPS and QZSS option 2

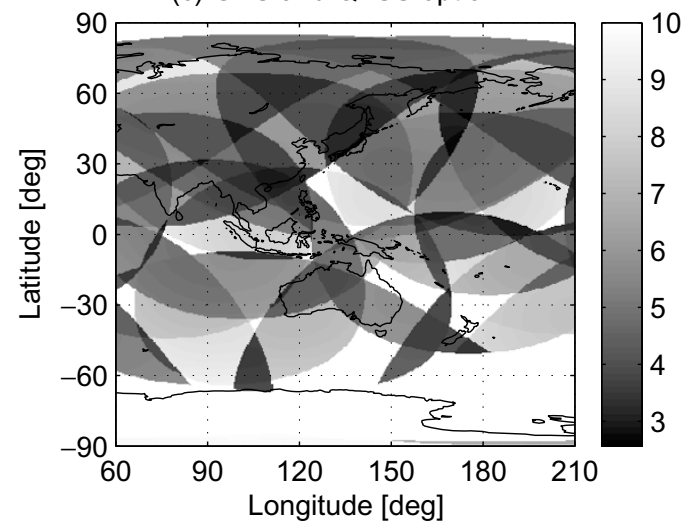

(b) GPS and QZSS option 1

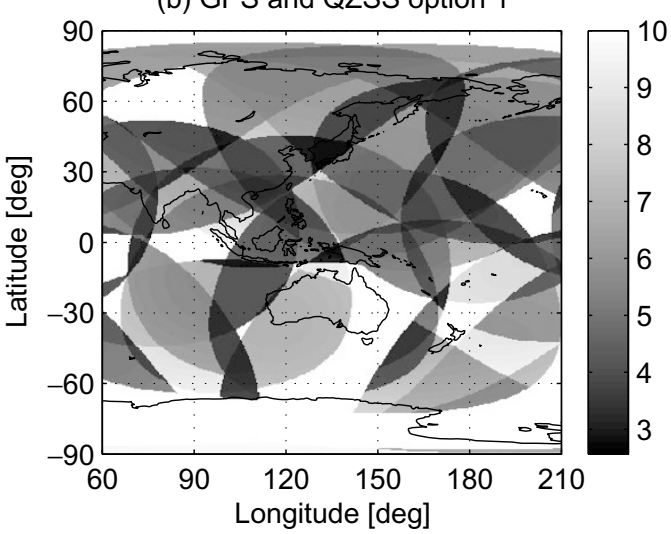

(d) GPS and QZSS option 3

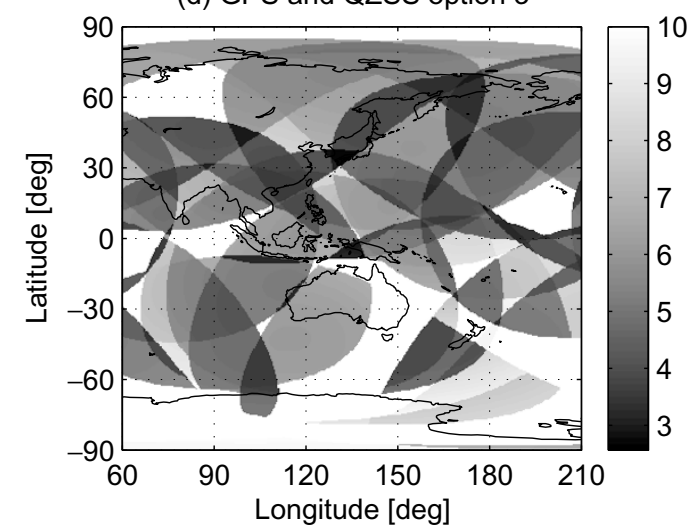

Fig. 4. Spatial variations of GDOP for different constellations.

(a) GPS only

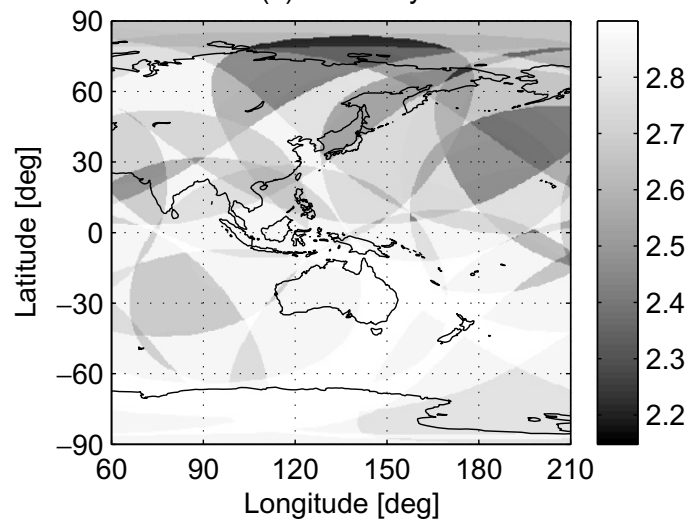

(c) GPS and QZSS option 2

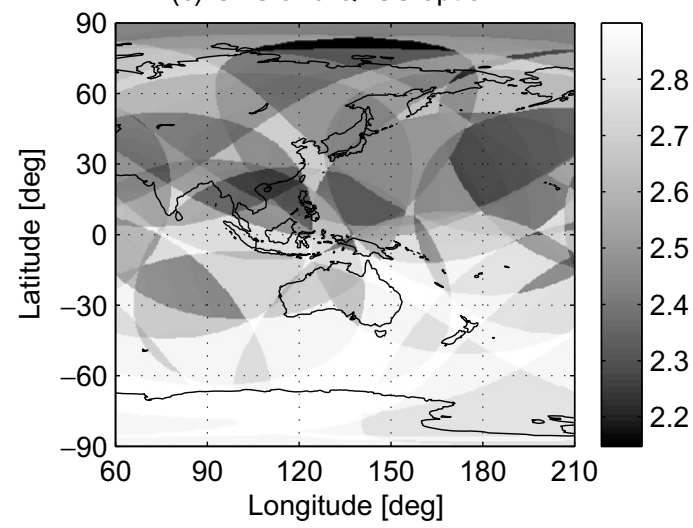

(b) GPS and QZSS option 1

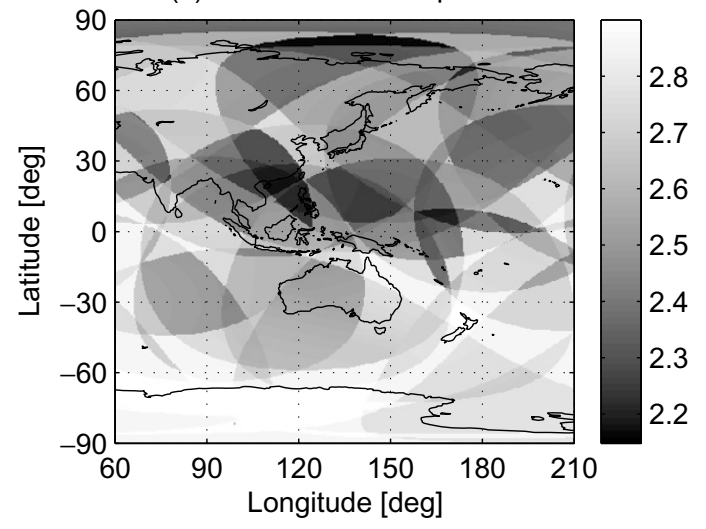

(d) GPS and QZSS option 3

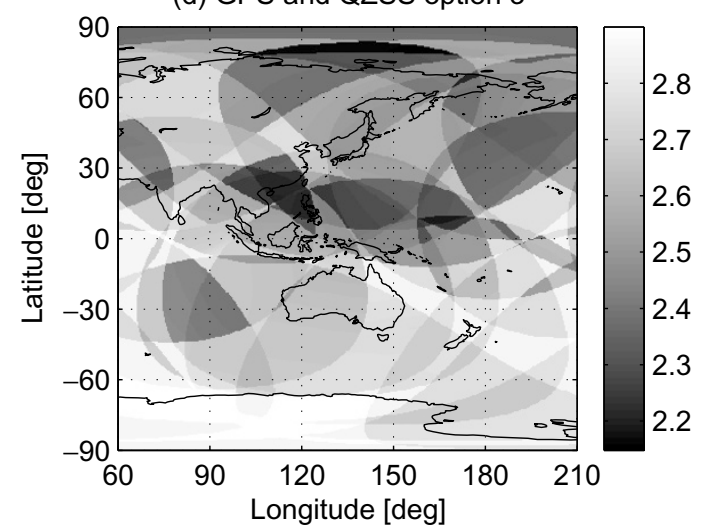

Fig. 5. Spatial variations of MDB for L1 code outlier for different constellations. 
(a) GPS only

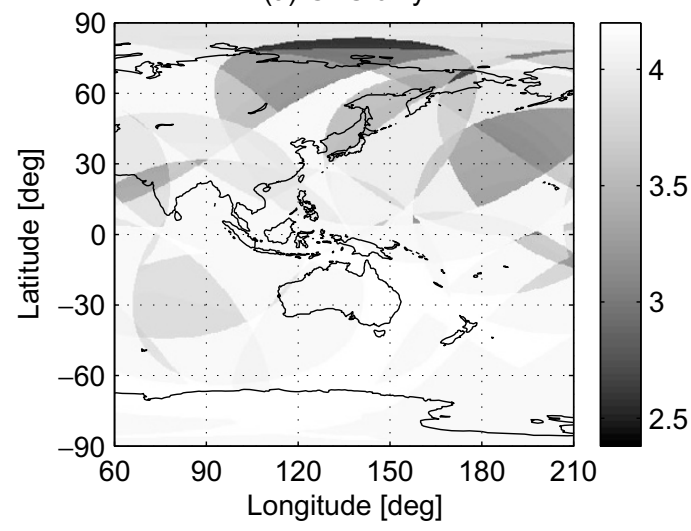

(c) GPS and QZSS option 2

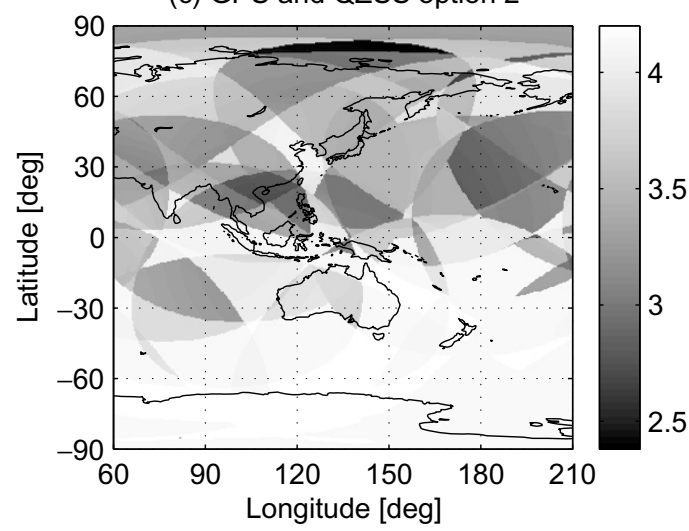

(b) GPS and QZSS option 1

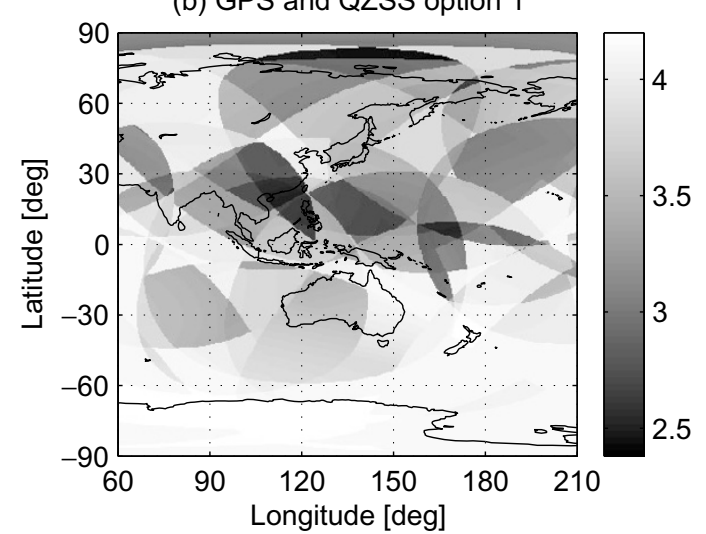

(d) GPS and QZSS option 3

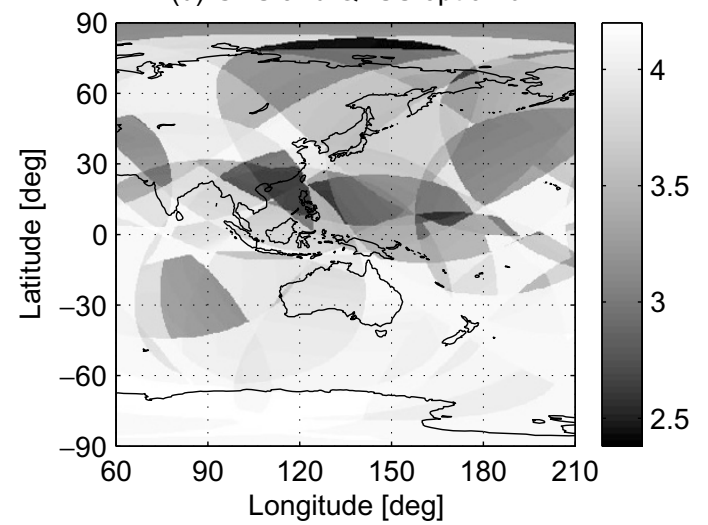

Fig. 6. Spatial variations of BNR for L1 code outlier for different constellations.

Table 5. Temporal variations of NVS, GDOP, MDB and BNR for L1 code outlier for different constellations at Tokyo (From Aug. 31, 2003, 00:00 to Sep. $6,2003,24: 00)$.

\begin{tabular}{|c|c|c|c|c|c|c|}
\hline \multicolumn{3}{|c|}{ System } & \multirow{2}{*}{$\begin{array}{c}\text { GPS only } \\
92.94 \%\end{array}$} & \multirow{2}{*}{$\begin{array}{c}\text { GPS+QZSS } 1 \\
100.00 \%\end{array}$} & \multirow{2}{*}{$\begin{array}{c}\text { GPS+QZSS } 2 \\
100.00 \%\end{array}$} & \multirow{2}{*}{$\frac{\text { GPS+QZSS } 3}{100.00 \%}$} \\
\hline Whole time & & & & & & \\
\hline \multirow{12}{*}{$\begin{array}{l}\text { Positioning } \\
\text { available time } \\
\text { (GPS only) }\end{array}$} & & MIN & 4 & 6 & 6 & 5 \\
\hline & NVS & MAX & 7 & 9 & 10 & 9 \\
\hline & & MEAN & 4.87 & 6.89 & 7.04 & 6.77 \\
\hline & & MIN & 2.94 & 2.55 & 2.55 & 2.52 \\
\hline & GDOP & MAX & 1637.15 & 20.06 & 18.74 & 26.46 \\
\hline & & MEAN & 11.51 & 4.98 & 5.28 & 5.10 \\
\hline & MDB & MIN & 2.38 & 2.24 & 2.19 & 2.21 \\
\hline & (L1 code & MAX & 2.86 & 2.72 & 2.72 & 2.77 \\
\hline & outlier) & MEAN & 2.74 & 2.54 & 2.50 & 2.54 \\
\hline & BNR & MIN & 3.14 & 2.72 & 2.48 & 2.66 \\
\hline & ( $\mathrm{L} 1$ code & MAX & 4.13 & 4.12 & 4.12 & 4.13 \\
\hline & outlier) & MEAN & 3.95 & 3.65 & 3.52 & 3.63 \\
\hline
\end{tabular}

2.70 , respectively. The average GDOP for GPS only is about 21.03, but the GDOP mean values of augmented GPS using the three QZSS options are about 11.90, 16.47 and 11.59, respectively for each constellation.

Figure 5 shows the spatial variations of MDB for L1 code outlier for the GPS only, and augmented GPS using the three QZSS options. The minimum MDB of GPS only is about
2.21, but augmented GPS using the three QZSS options give values 2.16, 2.15 and 2.15, respectively. The average MDB of GPS only is about 2.72, but the values of augmented GPS using the three QZSS options are about 2.57, 2.59 and 2.58, respectively.

Figure 6 shows the spatial variations of BNR for L1 code outlier for the GPS only and augmented GPS using the three 
(a) GPS only

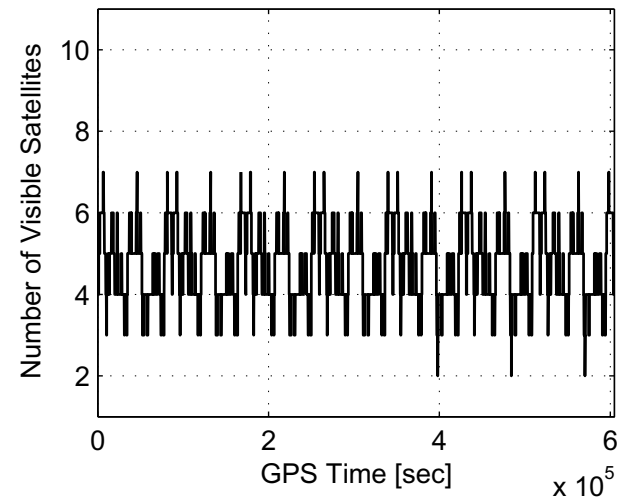

(c) GPS and QZSS option 2

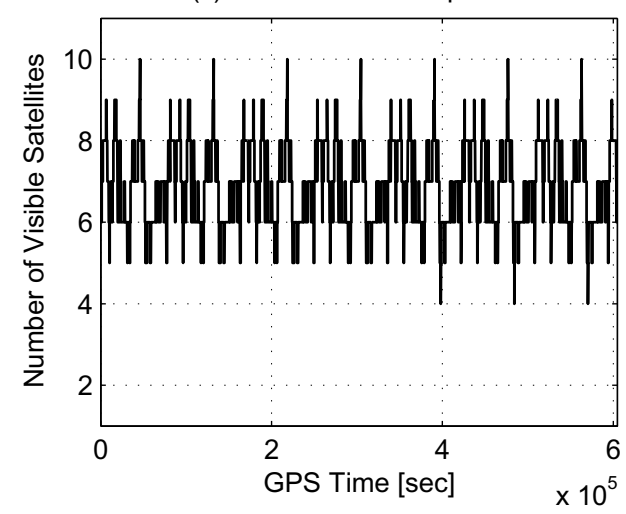

(b) GPS and QZSS option 1

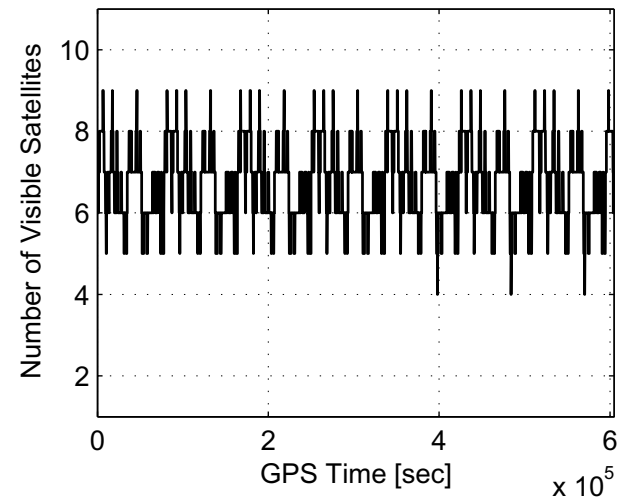

(d) GPS and QZSS option 3

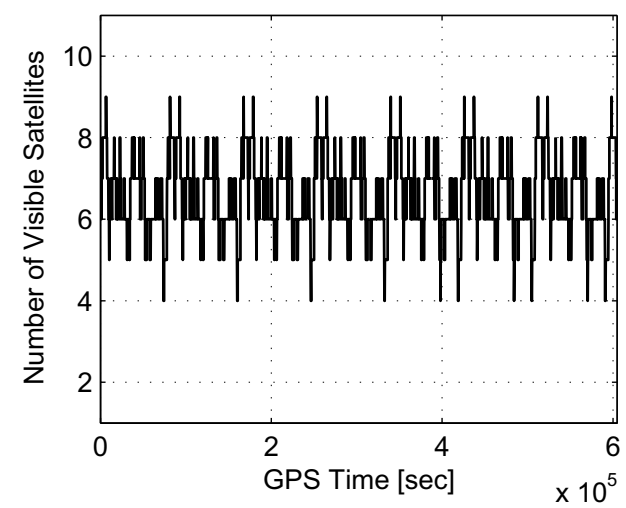

Fig. 7. Temporal variations of NVS for different constellations.

(a) QZSS option 1

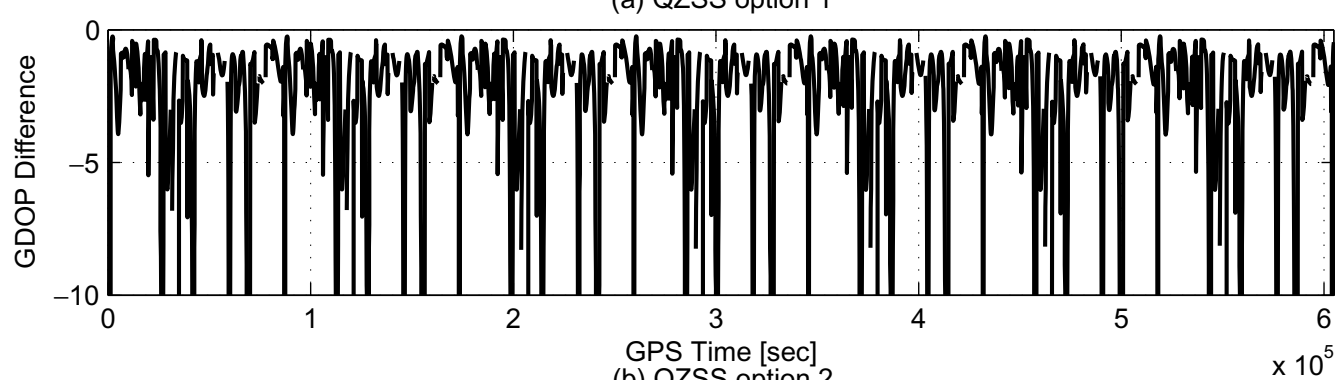

(b) QZSS option 2

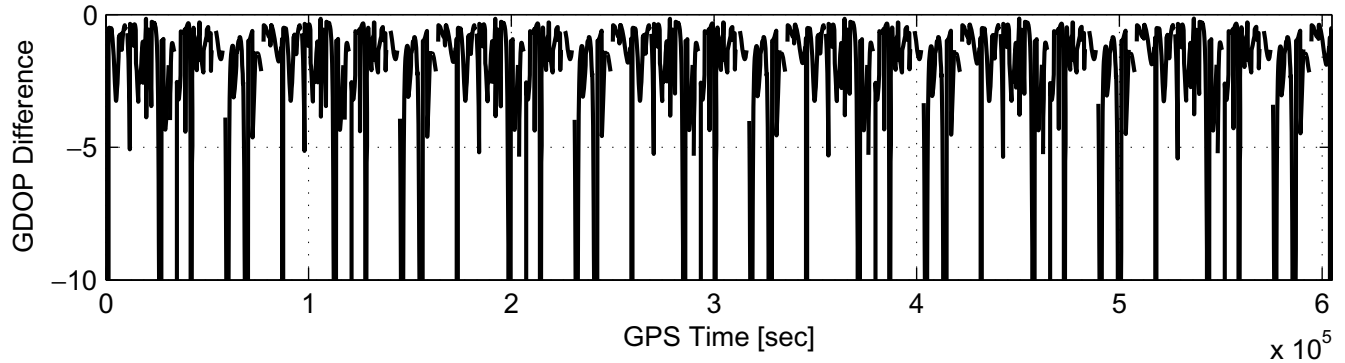

(c) QZSS option 3

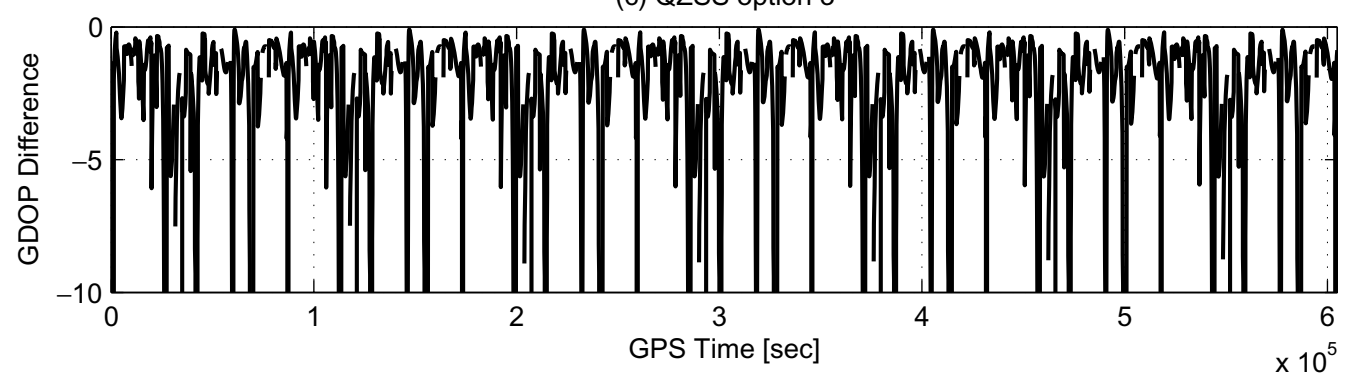

Fig. 8. Temporal variations of GDOP difference between having QZSS augmentation and not having it (three QZSS options). 
(a) QZSS option 1
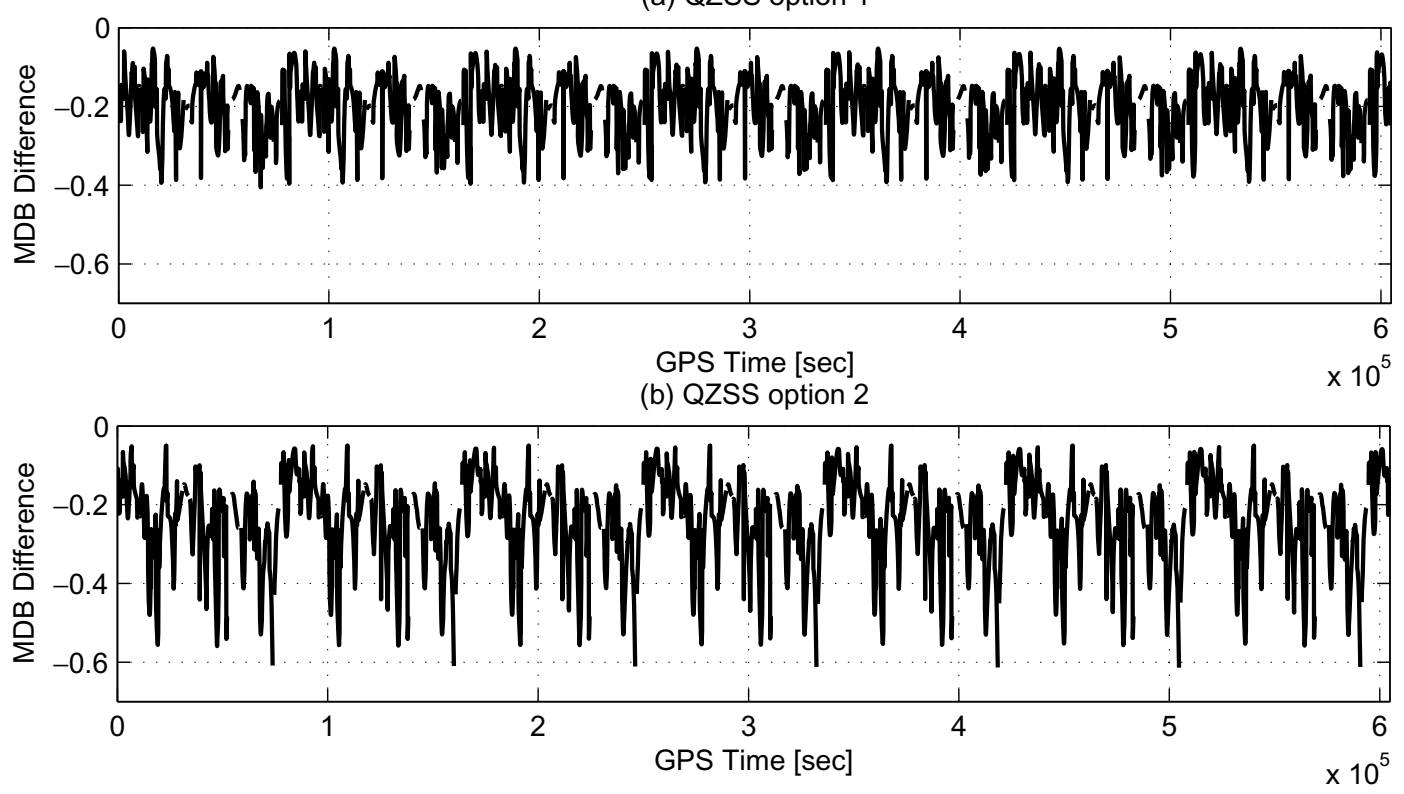

(c) QZSS option 3

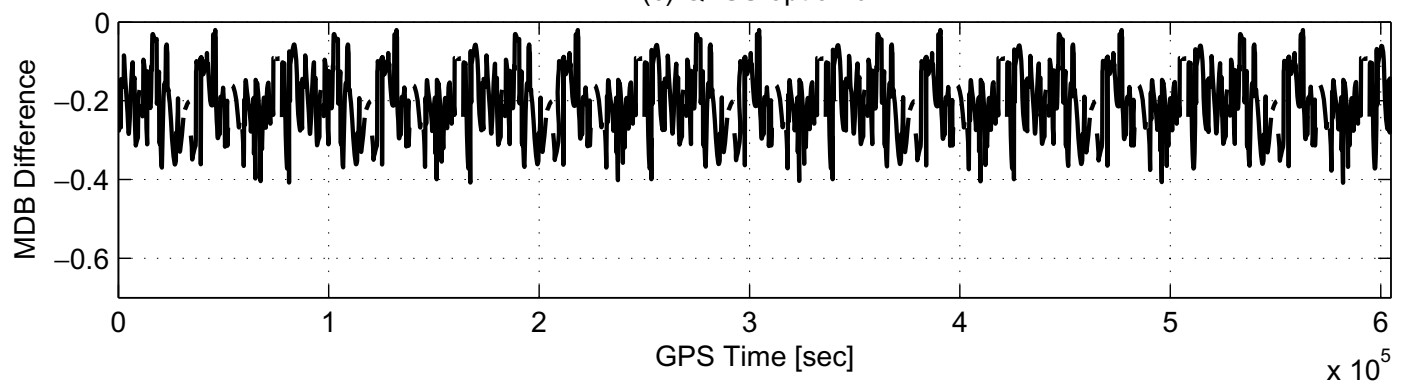

Fig. 9. Temporal variations of L1 code outlier MDB difference between having QZSS augmentation and not having it (three QZSS options).

QZSS options. The minimum BNR of GPS only is about 2.59, but augmented GPS using the three QZSS options give values $2.42,2.38$ and 2.40, respectively. The BNR mean value for GPS only is about 3.93, but the BNR mean values of augmented GPS using the three QZSS options are about 3.64, 3.66 and 3.67, respectively for each constellation.

It has been shown that any of the three QZSS options will not only extend the positioning available area, and improve the satellite visibility, and offer better GDOP, but also enhance the system reliability in Japan and its neighboring area. From the three QZSS options, QZSS option 3 can provide a little more favorable availability and accuracy than in the case of the two other QZSS options. But QZSS option 1 can provide a little more favorable availability, accuracy and reliability than in the case of QZSS option 2.

\subsection{Temporal Variations}

Table 5 summarizes the temporal variations of NVS, GDOP, MDB and BNR for L1 code outlier in the case of GPS only, and augmented GPS for the three QZSS options at Tokyo from August 31, 2003, 00:00 to September 6, 2003, 24:00. It is shown that with the augmentation by any of the three QZSS options, the time when positioning is available (NVS $\geq 4$ ) will be improved from $92.94 \%$ to $100.00 \%$. For temporal variations, augmentation using the three QZSS options can improve the positioning available time, but also enables some moments when have a very high GDOP, MDB and BNR. To analyze the performance of GPS augmentation using the three QZSS options, only the time when positioning is available in the case of GPS only is considered in this subsection.

Figure 7 shows the variation of NVS for the GPS only and augmented GPS using the three QZSS options over a one week period. The maximum NVS of GPS only is 7, but augmented GPS using the three QZSS options give values 9, 10 and 9, respectively. The average NVS of GPS only is about 4.87, but the values of augmented GPS using the three QZSS options are about 6.89, 7.04 and 6.77, respectively for each constellation.

The minimum GDOP of GPS only is about 2.94, but augmented GPS using the three QZSS options give vlues 2.55, 2.55 and 2.52, respectively. The GDOP mean values of GPS only and augmented GPS using the three QZSS options are $11.51,4.98,5.28$ and 5.10, respectively. Figure 8 shows the GDOP differences between having QZSS augmentation and not having it as a function of time.

The minimum MDB of GPS only is about 2.38, but the augmented GPS using the three QZSS options give values 2.24, 2.19 and 2.21, respectively. The MDB mean value of GPS only is about 2.74, but the MDB mean values of augmented GPS using the three QZSS options are about 2.54, 2.50 and 2.54, respectively. Figure 9 shows the temporal variations of L1 code outlier MDB difference between hav- 
(a) QZSS option 1
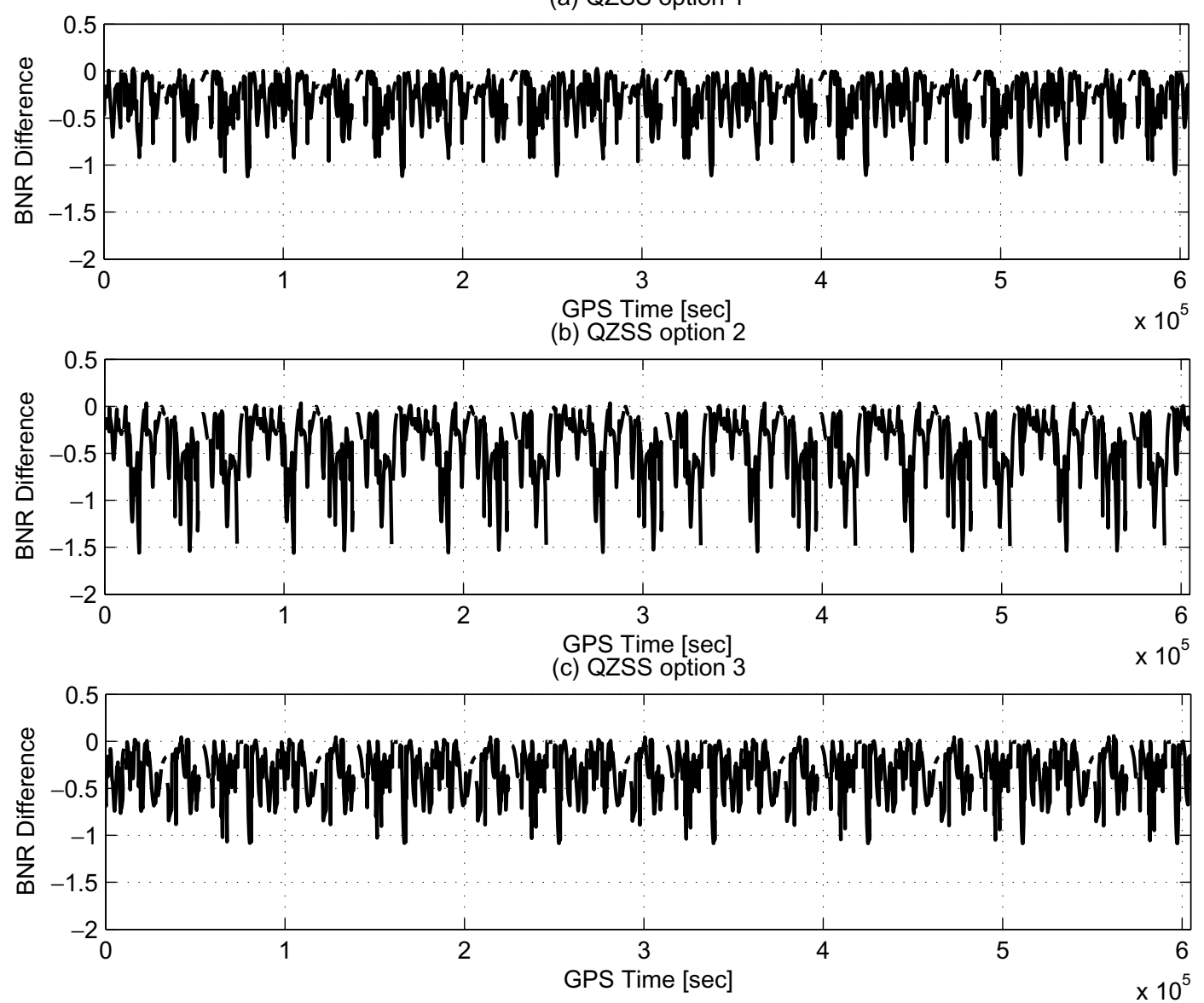

Fig. 10. Temporal variations of L1 code outlier BNR difference between having QZSS augmentation and not having it (three QZSS options).

ing QZSS augmentation and not having it.

The minimum BNR of GPS only is about 3.14, but the augmented GPS using the three QZSS options give values 2.72, 2.48 and 2.66, respectively. The BNR mean value of GPS only is about 3.95 , but the BNR mean values of augmented GPS using the three QZSS options are 3.65, 3.52 and 3.63, respectively. Figure 10 shows the temporal variations of the L1 code outlier BNR difference between having QZSS augmentation and not having it.

The results show that any of the three QZSS options will not only improve the positioning available time, and improve the satellite visibility, and offer better GDOP, but also will enhance the system reliability across Japan. From the three QZSS options, QZSS option 2 can provide a little more favorable availability and reliability than in case of the two other QZSS options, but QZSS option 1 can provide a little more favorable accuracy than in the case of the two other QZSS options.

\section{Conclusions}

This paper has focussed on the performance of GPS augmentation using the proposed Japanese QZSS. The QZSS satellite constellation and signal structure have been briefly introduced. The three single baseline models and stochastic model of GPS augmentation using QZSS have been analyzed. The measures for performance analysis, NVS, GDOP, MDB, MDE and BNR, have been described. The achievable performance of the GPS augmentation using QZSS are obtained using software simulation, and described by the spatial and temporal variations of NVS, GDOP, MDB and BNR. Three QZSS satellite constellation options have been investigated. It has been shown that QZSS does not only effectively improve the availability and accuracy of GPS positioning, but also enhances the reliability of GPS positioning in Japan and its neighboring area. From the three QZSS options, QZSS option 1 is the best satellite constellation option for Japan, although QZSS option 3 is the best satellite constellation option for the whole Asia-Pacific, Australian and New Zealand area.

Acknowledgments. The authors would like to acknowledge Dr. Tomoyuki Miyano, Tokyo Metropolitan College of Aeronautical Engineering, and Mr. Masayuki Saito, Advanced Space Business Corporation, Japan, for discussions on QZSS satellite constellation. The authors would also like to acknowledge Mr. Peter Joosten and Ms. Sandra Verhagen, Delft University of Technology, for software support.

\section{References}

de Jong, C. D., Minimal detectable biases of cross-correlated GPS observations, GPS Solutions, 3(3), 12-18, 2000.

FRP, Federal Radionavigation Plan, Final Report DOD-4650.5, United States Department of Defence and Department of Transportation, Washington, D.C. 20590, USA, 1999.

Kaplan, E. D., Understanding GPS-Principles and Applications, Artech 
House, Boston, 1996.

Kawano, I., System Study of Next Generation Satellite Positioning System, SANE 98-144, Institute of Electronics, Information and Communication Engineers, 1999 (in Japanese).

Kawano, I., Satellite Positioning System Using Quasi-Zenith and Geostationary Satellites, The transactions of the Institute of Electronics, Information and Communication Engineers, J84-B:2092-2100, 2001.

Kimura, K. and M. Tanaka, Inclined Geo-synchronous Orbit Constellations Suitable to Fixed Satellite Communications, SANE 99-123, Institute of Electronics, Information and Communication Engineers, 2000 (in Japanese).

Kogure, S. and I. Kawano, GPS Augmentation and Complement Using Quasi-Zenith Satellite System (QZSS), Proceeding of the 21st AIAA International Communications Satellite Systems, Yokohama, Japan, 2003.

Kon, M., System Overview and Applications of Quasi-Zenith Satellite Systems, Proceeding of the 21st AIAA International Communications Satellite Systems, Yokohama, Japan, 2003,

Murotani, M., S. Urasaki, and O. Yamanaka, Quasi-geostationary orbit and application in communication, broadcast and positioning, Research on Satellite Communication, 101, 1-77, 2003 (in Japanese).

O'Keefe, K., Availability and Reliability Advantages of GPS/Galileo Integration, Proceeding of the 14th International Technical Meeting of the Satellite Division of the Institute of Navigation (ION GPS-2001), pp. 2096-2104, Salt Lake City, UT, USA, 2001.

Petrovski, I. G., M. Ishii, H. Torimoto, H. Kishimoto, T. Furukawa, M. Saito, T. Tanaka, and H. Maeda, QZSS_-Japan's new integrated communication and positioning service for mobile users, GPS World, 14(6), 24-29, 2003.

Shaw, M., D. A. Turner, and K. Sandhoo, Modernization of the Global Positioning System, Proceeding of the Japanese Institute of Navigation, GPS Symposium 2002, pp. 3-12, Tokyo, Japan, 2002.

Takahashi, M., K. Kimura, and M. Tanaka, An Adaptability Study of QuasiZenith Satellite Orbits for Land Mobile Satellite Communications, SANE 99-31, Institute of Electronics, Information and Communication Engineers, 1999 (in Japanese).

Teunissen, P. J. G., Internal reliability of single frequency GPS data, Artifical
Satellite, 32(2), 63-73, 1997.

Teunissen, P. J. G., Minimal detectable biases of GPS data, Journal of Geodesy, 72(4), 236-244, 1998.

Teunissen, P. J. G. and C. D. de Jong, Reliability of GPS Cycle Slip and Outlier Detection, Proceeding of INSMAP98, pp. 78-86, Melbourne, Florida, 1998.

Teunissen, P. J. G. and A. Kleusberg, GPS for Geodesy, second edition, Springer, 1998.

Teunissen, P., P. Joosten, and C. Tiberius, A Comparison of TCAR, CIR and LAMBDA GNSS Ambiguity Resolution, Proceeding of the 15th International Technical Meeting of the Satellite Division of the Institute of Navigation (ION GPS-2002), pp. 2799-2808, Portland, OR, USA, 2002.

Tiberius, C., T. Pany, B. Eissfeller, K. de Jong, P. Joosten, and S. Verhagen, Integral GPS-Galileo Ambiguity Resolution, ENC-GNSS 2002 PROCEEDINGS, Copenhagen, Denmark, 2002.

Verhagen, S., Internal and External Reliability of an Integrated GNSSPseudolite Positioning System, Proceeding of the 2nd Symposium on Geodesy for Geotechnical and Structural Engineering, pp. 431-441, Berlin, 2002a.

Verhagen, S., Performance Analysis of GPS, Galileo and Integrated GPSGalileo, Proceeding of the 15th International Technical Meeting of the Satellite Division of the Institute of Navigation (ION GPS-2002), pp. 2208-2215, Portland, OR, USA, 2002b.

Verhagen, S. and P. Joosten, Algorithms for Design Computations for Integrated GPS-Galileo, Proceedings of the European Navigation Conference (ENC-GNSS 2003), Graz, Austria, 2003.

Yamamoto, S. and K. Kimura, A Study on a Satellite Communications System for Polar Regions Using Quasi-Zenithal Satellites, Proceeding of the 21st AIAA International Communications Satellite Systems, Yokohama, Japan, 2003.

F. Wu (e-mail: fwu@e.kaiyodai.ac.jp), N. Kubo, and A. Yasuda 